\title{
Improved health-related quality of life in patients treated with topical sirolimus for facial angiofibroma associated with tuberous sclerosis complex
}

Takashi Hatano ( $\nabla$ hatano-t@jreast.co.jp )

JR Tokyo General Hospital

Yuki ohno

JR Tokyo General Hospital

YU Imai

JR Tokyo General Hospital

Jun Moritake

JR Tokyo General Hospital

Katsuhisa Endo

JR Tokyo General Hospital

Mayumi Tamari

The Jikei University School of Medicine

Shin Egawa

The Jikei University School of Medicine

\section{Research}

Keywords: tuberous sclerosis complex, facial angiofibroma, topical sirolimus, health-related quality, of life, mental health

Posted Date: April 1st, 2020

DOI: https://doi.org/10.21203/rs.3.rs-20090/v1

License: (c) (1) This work is licensed under a Creative Commons Attribution 4.0 International License.

Read Full License

Version of Record: A version of this preprint was published at Orphanet Journal of Rare Diseases on June 1st, 2020. See the published version at https://doi.org/10.1186/s13023-020-01417-5. 


\section{Abstract}

Background : Tuberous sclerosis complex (TSC) is a rare autosomal dominant disorder forming hamartomas throughout the body. Facial angiofibromas (FAs) occur in $75 \%$ of TSC patients, which are often enlarged, impairing the appearance of the face, and reducing the patient's quality of life (QOL). The aim of this study was to characterize the impact of topical sirolimus treatment on the health-related QOL in patients with FA associated with TSC.

Methods : We investigated a total of 33 patients who received sirolimus gel treatment for FA associated with TSC and assessed the changes in the health-related QOL using the Medical Outcomes Study 36-Item Short Form (SF-36) Health Survey. SF-36 surveys were performed before and after three months of treatment. The conditions of the patients after using the sirolimus gel were categorized into the following three categories: "improved," "unchanged," and "aggravated." Adverse events were investigated using the CTCAE v5.0-JCOG.

Results : After 3 months of sirolimus gel treatment, three scale scores of the SF-36, vitality (VT), social function (SF), and mental health (MH), were significantly improved compared to before the treatment. The VT and SF in patients who had improved FA were significantly better than those in the other patients. There were no significant differences in any scale scores between patients with and without adverse events at three months after the initiation of sirolimus gel treatment.

Conclusions : This is the first report regarding improved health-related quality of life in patients treated with sirolimus gel for FA associated with TSC by using the SF-36. The three scale scores associated with mental health were significantly improved compared to before the treatment. The health-related QOL in patients receiving sirolimus gel treatment is more strongly affected by the treatment efficacy than adverse events.

\section{Background}

Tuberous sclerosis complex (TSC) is a rare autosomal dominant inherited disorder characterized by systemic hamartomas and hypomelanotic macules ${ }^{1}$. It is caused by genetic mutations of either the TSC1 gene on chromosome 9 or the TSC2 gene on chromosome 16, which encode hamartin and tuberin, respectively 2,3 . Dysfunction of hamartin or tuberin induces the constitutive activation of mammalian target of rapamycin complex 1 (mTORC1) ${ }^{4}$. Most symptoms of TSC are considered to be derived from the activation of mTORC1. Since approximately $75 \%$ of patients with TSC suffer from facial angiofibromas (FAs), which are often enlarged, impairing the appearance of the face, and reducing the patient's quality of life (QOL), patients with FAs need to be treated.

Wataya-Kaneda et al. reported phase 2 and 3 randomized clinical trials of topical sirolimus treatment with a sirolimus gel formulation in TSC patients and showed significant reductions in the size and color of the FAs associated with TSC ${ }^{6,7}$. In Japan, sirolimus gel was approved for the treatment of TSC skin 
lesions in 2018. However, few studies that assess the effect of topical sirolimus treatment on the healthrelated QOL in patients with FA associated with TSC have been conducted.

In the present study, we evaluated the changes in the health-related QOL of patients receiving sirolimus gel treatment for FA associated with TSC, using the Medical Outcomes Study 36-Item Short Form (SF-36) Health Survey, one of the most widely used surveys for health-related QOL ${ }^{8}$.

\section{Methods}

\section{Study design and patients}

FA associated with TSC was diagnosed based on the International Tuberous Sclerosis Complex Consensus Conference (ITSCCC) diagnostic criteria after consultations with an internist and dermatologist. A total of 33 patients met the diagnostic criteria and received topical sirolimus (Raparimus ${ }^{\circledR}$ gel; Nobelpharma, Tokyo, Japan). The sirolimus gel contained $0.2 \%$ sirolimus and additives including alcohol. Each patient was instructed to spread $400 \mathrm{mg}$ of the sirolimus gel evenly on the FA site twice a day. The conditions of the patients after three months of treatment with sirolimus gel were classified into three categories according to the criteria shown in Table 1: "improved," "unchanged," and "aggravated." 7 Adverse events were investigated using the CTCAE v5.0-JCOG (National Cancer Institute, Bethesda, MD, USA). This study was approved by the institutional review board of JR Tokyo General Hospital (No. H29-27).

Table 1

The criteria for improvements in facial angiofibroma lesions from baseline

\begin{tabular}{|lll|}
\hline Rating & Size & Color \\
\hline Improved & Reduced in $\geq 50 \%$ of lesions & $\begin{array}{l}\text { Improved by } \geq 2 \text { reddishness levels in } \geq \\
50 \% \text { of lesions }\end{array}$ \\
\cline { 1 - 2 } Unchanged & Not obviously changed & Not obviously changed \\
Aggravated & $\begin{array}{l}\text { Increased or newly formed papules in } \geq \\
50 \% \text { of lesions }\end{array}$ & $\begin{array}{l}\text { Aggravated by } \geq 2 \text { reddishness levels in } \geq \\
50 \% \text { of lesions }\end{array}$ \\
\hline
\end{tabular}

\section{Exclusion Criteria}

The following patients were excluded: those with erosions, ulcers, or other skin lesions associated with FAs, uncontrollable epileptic seizures despite being treated with anti-epileptic agents, poor respiratory condition due to lung lymphangioleiomyomatosis, or systemic treatment with an mTOR inhibitor; and those who were pregnant or who could not periodically visit the hospital. 


\section{Assessment Of The Health-related Qol}

For assessment of the health-related QOL in 33 patients, the Japanese version 2 of the SF-36 was used ${ }^{8}$. This questionnaire is completed by patient self-report through survey or interview, and consists of 36 selfadministered questions that quantify the health-related QOL using 8 multi-item scales for the health status covering both the mental and physical aspects of health, namely the physical function (PF), role limitations because of physical health problems (RP), bodily pain (BP), general health perception (GH), vitality (VT), social function (SF), role limitations because of emotional problems (RE), and mental health $(\mathrm{MH})$. The PF, RP, BP, GH and VT indicate physical health, and the GH, VT, SF, RE and MH indicate mental health. Each domain is scored on a scale of 0 to 100, with lower scores indicating a poorer health status. We standardized these domain scores using Japanese population norms to give mean scores of 50 and standard deviations of $10^{8,9}$. In this study, SF-36 surveys were performed before and at three months after the initiation of sirolimus gel treatment.

\section{Statistical Analysis}

The SF-36 scores are presented as the mean and the standard deviation. The pre- and post-treatment scores were analyzed using unpaired t-tests. A P level of $<0.05$ was considered to be statistically significant.

\section{Results}

\section{Patient characteristics, treatment efficacy and adverse events}

We analyzed 33 patients with FA associated with TSC who received sirolimus gel treatment (Table 2). The median age of the patients was 25 (range 14-55) years. The performance status was 2 in one patient who showed muscle weakness. Sirolimus gel treatment improved FA associated with TSC in 23 of the 33 $(70 \%)$ patients after 3 months of treatment. However, the conditions of the remaining $10(30 \%)$ were unchanged. None of the conditions of these patients were classified as "aggravated." Adverse events related to sirolimus gel were observed in $12(36 \%)$ patients. The major adverse events were acne, application site irritation, dry skin, and pruritus. There were no cases of grade $\geq 3$ adverse events. Table 3 comparess the treatment-related adverse events of the improved group and the unchanged group. All patients' events improved with symptomatic treatment. None of the patients discontinued the treatment due to adverse events. 
Table 2

Baseline characteristics of 33 patients receiving sirolimus gel for facial angiofibroma associated with tuberous sclerosis complex

\begin{tabular}{|lll|}
\hline Characteristics & Number & $\%$ \\
\hline Median age (range) & 25 (14-55) & \\
\hline Sex & & \\
\hline Male/Female & $17 / 16$ & \\
\hline ECOG performance status & & \\
\hline 0/1/2 & $24 / 8 / 1$ & \\
\hline Major features & & 61 \\
\hline$\geq 3$ Hypomelanotic macules & 20 & 55 \\
\hline$\geq 2$ Ungual fibromas & 18 & 52 \\
\hline Shagreen patch & 17 & 18 \\
\hline Multiple retinal hamartomas & 6 & 76 \\
\hline Cortical dysplasias & 25 & 58 \\
\hline Subependymal nodules & 19 & 15 \\
\hline Subependymal giant cell astrocytoma & 5 & 12 \\
\hline Cardiac rhabdomyoma & 4 & 18 \\
\hline Lung lymphangioleiomyomatosis & 6 & 94 \\
\hline$\geq 2$ Renal angiomyolipomas & 31 & \\
\hline Minor features & 10 & \\
\hline$\geq 3$ Dental enamel pits & 6 & \\
\hline$\geq 2$ Intraoral fibromas & 5 & \\
\hline Retinal achromic patch & & \\
\hline Multiple renal cysts & & \\
\hline Nonrenal hamartomas & & \\
\hline
\end{tabular}


Table 3

The incidence rates of treatment-related adverse events

\begin{tabular}{|lll|}
\hline Event & $\begin{array}{l}\text { Improved group } \\
(\mathbf{n}=\mathbf{2 3})\end{array}$ & $\begin{array}{l}\text { Unchanged group } \\
(\mathbf{n}=10)\end{array}$ \\
\hline Acne & $2(9 \%)$ & $2(20 \%)$ \\
\hline Application site irritation & $2(9 \%)$ & $1(10 \%)$ \\
\hline Dry skin & $3(13 \%)$ & $0(0 \%)$ \\
\hline Pruritus & $1(4 \%)$ & $1(10 \%)$ \\
\hline
\end{tabular}

\section{Evaluation Of The Health-related Qol}

We evaluated the changes in the health-related QOL in patients with sirolimus gel treatment for FA associated with TSC by using the SF-36. After 3 months of sirolimus gel treatment, three scale scores of the SF-36 (VT, SF, and MH) were significantly improved compared to before treatment (Fig. 1). For the remaining five items, there were no marked differences after treatment compared with before treatment.

We then assessed the influences of the therapeutic efficacy and adverse events of sirolimus gel treatment on the health-related QOL. Figure 2 shows a comparison of the scale scores of the SF-36 three months after the initiation of sirolimus gel treatment for the improved group and the unchanged group. There were significant differences in two scale scores (VT and SF) between these two groups. Figure 3 shows the distribution in the difference between the scale scores of each group before and after three months of the treatment. The VT and SF score improvement rates in the improved group were 78\% (18/23) and 74\% $(17 / 23)$, respectively, and only 3 patients had decreased scores on both scales. Figure 4 shows that there were no significant differences in any scale scores of the SF-36 between patients with and without adverse events after three months of sirolimus gel treatment.

\section{Discussion}

TSC induces the development of various hamartomas throughout the body, including angiomyolipomas and angiofibromas. These tumors are responsible for the inhibition of mTOR. Sirolimus gel, an mTOR inhibitor, is effective against FA associated with TSC. In the UK management guidelines, topical mTOR inhibitors are recommended as the first line treatment for skin lesions associated with TSC ${ }^{1}$.

To date, several studies have reported the influence of topical mTOR inhibitors treatment on patients' QOL. Wataya-Kaneda et al. assessed the QOL improvement of patients with sirolimus gel treatment for FA associated with TSC using the Dermatology Life Quality Index (DLQI) and Children's DLQI (CDLQI). They reported that the total scores on the DLQI and CDLQI after 12 weeks of treatment did not differ markedly between the sirolimus gel group and the placebo group ${ }^{7}$. Similar findings were reported in 
previous studies analyzing the QOL improvement of patients with topical mTOR inhibitors for FA associated with TSC using the DLQI and CDLQI ${ }^{10,11}$. The DLQI and CDLQI are often used for QOL assessments in the treatment of skin diseases $7,12,13$. They are simple and comprised of 10 questions evaluating the disease impact on key aspects of patients' lives ${ }^{12-14}$. However, there are few questions related to vitality and mental health, so the DLQI and CDLQI are not able to evaluate the mental health of patients sufficiently.

In this study, we used the SF-36 health survey to prospectively assess the changes in the physical and mental health of Japanese patients who had FA associated with TSC and were treated with sirolimus gel. The three scale scores associated with mental health, VT, SF, and $\mathrm{MH}$, were significantly improved compared to before the treatment.

FAs influence the mental status of TSC patients and their families ${ }^{11}$. Since FAs often cause disfigurement, patients personality can become introverted, with reduced sociability ${ }^{11}$. Therefore, the treatment of FA might improve not only skin lesions but also mental health.

We further assessed the treatment efficacy and adverse events. It has been recognized that these two major factors are directly associated with patient satisfaction and health-related QOL during treatment. Patients in whom FA improved had significantly higher mental health scale scores for VT and SF than those whose condition did not improve. Furthermore, there was no marked influence of adverse events related to sirolimus gel treatment on the health-related QOL. These findings suggested that mental health became better in patients whose FA improved after administration of sirolimus gel. In this study, the adverse events with sirolimus gel treatment were tolerable, with only low-grade severity.

However, our study had several limitations. First, several surveys for the evaluation of health-related QOL have been developed, including as the Sickness Impact Profile (SIP) and the Functional Assessment of Cancer Therapy-General (FACT-G) ${ }^{15,16}$. In the present study, we evaluated the health-related QOL using only the SF-36. Further studies are required using other surveys for the evaluation of health-related QOL. Second, this study did not monitor patients over a long period. The long-term safety and tolerability of sirolimus gel treatment have not been sufficiently investigated. Thus, further studies on the influence of long-term sirolimus gel treatment upon health-related $\mathrm{QOL}$ are required.

\section{Conclusions}

We evaluated the changes in the health-related QOL in patients with sirolimus gel treatment for FA associated with TSC by using the SF-36. Three scale scores associated with mental health, VT, SF, and $\mathrm{MH}$, were significantly improved compared to before the treatment. Furthermore, improvements of the VT and SF scores were observed in the improved group compared with the unchanged group. However, further investigations are necessary to achieve a better understanding of the efficacy and safety of sirolimus gel treatment for FA associated with TSC 


\section{Abbreviations}

QOL: quality of life; FA: facial angiofibroma; TSC: tuberous sclerosis complex; SF-36: Medical Outcomes Study 36-Item Short Form; mTORC1: mammalian target of rapamycin complex 1; ITSCCC: International Tuberous Sclerosis Complex Consensus Conference; PF: physical function; RP: role limitations because of physical health problems; BP: bodily pain; GH: general health perception; VT: vitality; SF: social function; RE: role limitations because of emotional problems; $\mathrm{MH}$ : and mental health; DLQI: Dermatology Life Quality Index; CDLQI: Children's DLQI; SIP: Sickness Impact Profile; FACT-G: Functional Assessment of Cancer Therapy-General;

\section{Declarations}

\section{Acknowledgments}

We thank the patients and their families for participating.

\section{Authors' contributions}

TH participated in the design of the study and drafted the manuscript. YI, JM and KE participated in data acquisition. MT performed the statistical analysis. SE participated in the design of the study and helped to write the paper. All authors read and approved the final manuscript.

\section{Funding}

No external funding.

\section{Availability of data and materials}

Data are available on reasonable request.

\section{Ethics approval and consent to participate}

This study was approved by the institutional review board of JR Tokyo General Hospital (No. H29-27). All patients provided written informed consent before entering the study. 


\section{Consent for publication}

Not applicable.

\section{Competing interests}

The authors of this article declare no conflict of interest.

\section{Author details}

${ }^{1}$ Tuberous Sclerosis Complex Unit, JR Tokyo General Hospital. ${ }^{2}$ Department of Dermatology, JR Tokyo General Hospital. ${ }^{3}$ Department of Urology, JR Tokyo General Hospital. ${ }^{4}$ Research Center for Medical Science, Division of Molecular Genetics, The Jikei University School of Medicine. ${ }^{5}$ Department of Urology, The Jikei University School of Medicine.

\section{References}

1. Amin S, Kingswood JC, Bolton RF, et al. (2019) The UK guidelines for management and surveillance of tuberous sclerosis complex. QJM 112:171-182.

2. van Slegtenhorst $M$, de Hoogt $R$, Hermans $C$, et al. (1997) Identification of the tuberous sclerosis gene TSC1 on chromosome 9q34. Science 277:805-808.

3. The European Chromosome 16 Tuberous Sclerosis Consortium. (1993) Identification and characterization of the tuberous sclerosis gene on chromosome 16. Cell 75:1305-1315.

4. Tee AR, Manning BD, Roux PP, et al. (2003) Tuberous sclerosis complex gene products, tuberin and hamartin, control mTOR signaling by acting as a GTPase-activating protein complex toward Rheb. Curr Biol 13:1259-1268.

5. Krueger DA, Northrup H, International Tuberous Sclerosis Complex Consensus Group. (2013) Tuberous sclerosis complex surveillance and management: recommendations of the 2012 International Tuberous Sclerosis Complex Consensus Conference. Pediat Neurol 48: 255-265.

6. Wataya-Kaneda M, Nakamura A, Tanaka M et al. (2017) Efficacy and safety of topical sirolimus therapy for facial angiofibromas in the tuberous sclerosis complex: a randomized clinical trial. JAMA Dermatol 153:39-48.

7. Wataya-Kaneda M, Ohno Y, Fujita Y et al. (2018) Sirolimus gel treatment vs placebo for facial angiofibromas in patients with tuberous sclerosis complex: a randomized clinical traial. JAMA Dermatol 154:781-788.

8. Fukuhara S, Bito S, Green J et al. (1998) Translation, adaptation, and validation of the SF-36 Health Survey for use in Japan. J Clin Epidemiol 51:1037-1044. 
9. Fukuhara S, Ware JE Jr, Koshinski M et al. (1998) Translation, adaptation, and validation of the SF36 Health Survey for use in Japan. J Clin Epidemiol 51:1045-1053.

10. Koenig MK, Bell CS, Hebert AA et al. (2018) Efficacy and safety of topical rapamycin in patients with facial angiofibromas secondary to tuberous sclerosis complex. 154:773-780.

11. Crall C, Valle M, Kapur K et Al. (2016) Effect of angiofibromas on quality of life and access to care in tuberous sclerosis patients and their caregivers. Pediatr Dermatol 33:518-525.

12. Finlay AY, Khan GK. (1994) Dermatology Life Quality Index (DLQI) - a simple practical measure for routine clinical use. Clin Exp Dermatol 19:210-216.

13. Lewis-Jones MS, Finlay AY. (1995) The children's Dermatology Life Quality Index (DLQI): initial validation and practical use. Br J Dermatol 132:942-949.

14. Takahashi N, Suzukamo Y, Nakamura M et al. (2006) Japanese version of the Dermatology Life Quality Index: validity and reliability in patients with acne. Health Qual Life Outcomes 4:46.

15. Bergne M, Bobbitt RA, Carter WB et al. (1981) The Sickness Impact Profile: development and final revision of a health status measure. Med Care 19:787-805.

16. Cella DF, Tulsky DS, Gray G, et al. (1993) The Functional Assessment of Cancer Therapy scale: development and validation of the general measure. J Clin Oncol. 11:570-579.

\section{Figures}

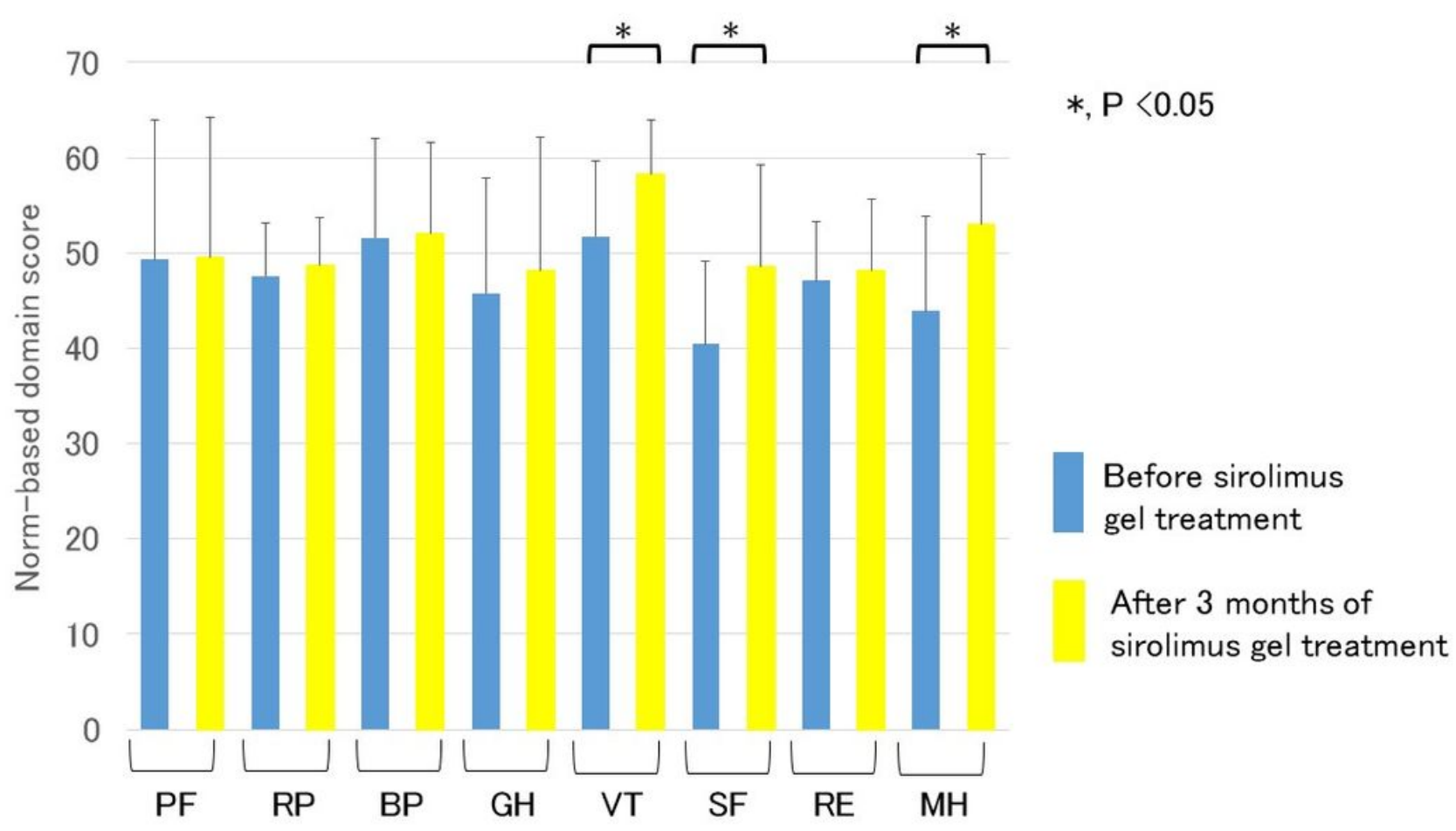

Figure 1 
Comparison of the scale scores on the Medical Outcomes Study 36-Item Short Form in patients with sirolimus gel treatment for facial angiofibroma with tuberous sclerosis complex before and after three months of treatment. PF, physical function; RP, role limitations because of physical health problems; BP, bodily pain; $\mathrm{GH}$, general health perception; VT, vitality; SF, social function; RE, role limitations because of emotional problems; $\mathrm{MH}$, mental health. ${ }^{*}, \mathrm{P}<0.05$. The scale scores of the $\mathrm{VT}, \mathrm{SF}$, and $\mathrm{MH}$ were significantly improved compared to before the treatment.

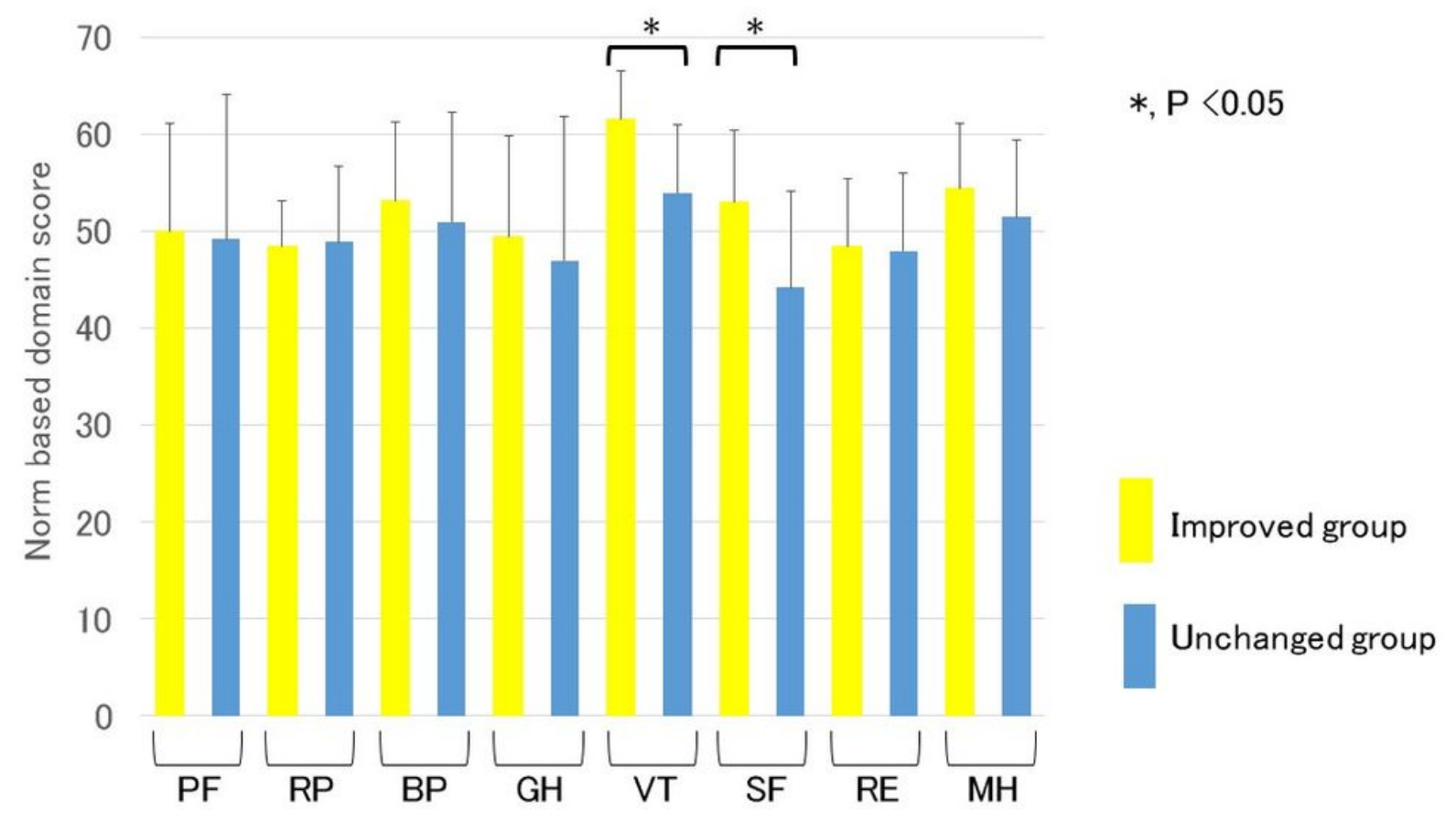

\section{Figure 2}

Comparison of the scale scores on the Medical Outcomes Study 36-Item Short Form three months after the initiation of sirolimus gel treatment for facial angiofibroma associated with tuberous sclerosis complex between patients with and without improved facial angiofibroma. PF, physical function; RP, role limitations because of physical health problems; BP, bodily pain; $\mathrm{GH}$, general health perception; VT, vitality; SF, social function; RE, role limitations because of emotional problems; $\mathrm{MH}$, mental health. * $\mathrm{P}$ $<0.05$. The scale scores of the VT and SF in patients whose FA had improved were significantly higher than those whose condition did not improve. 


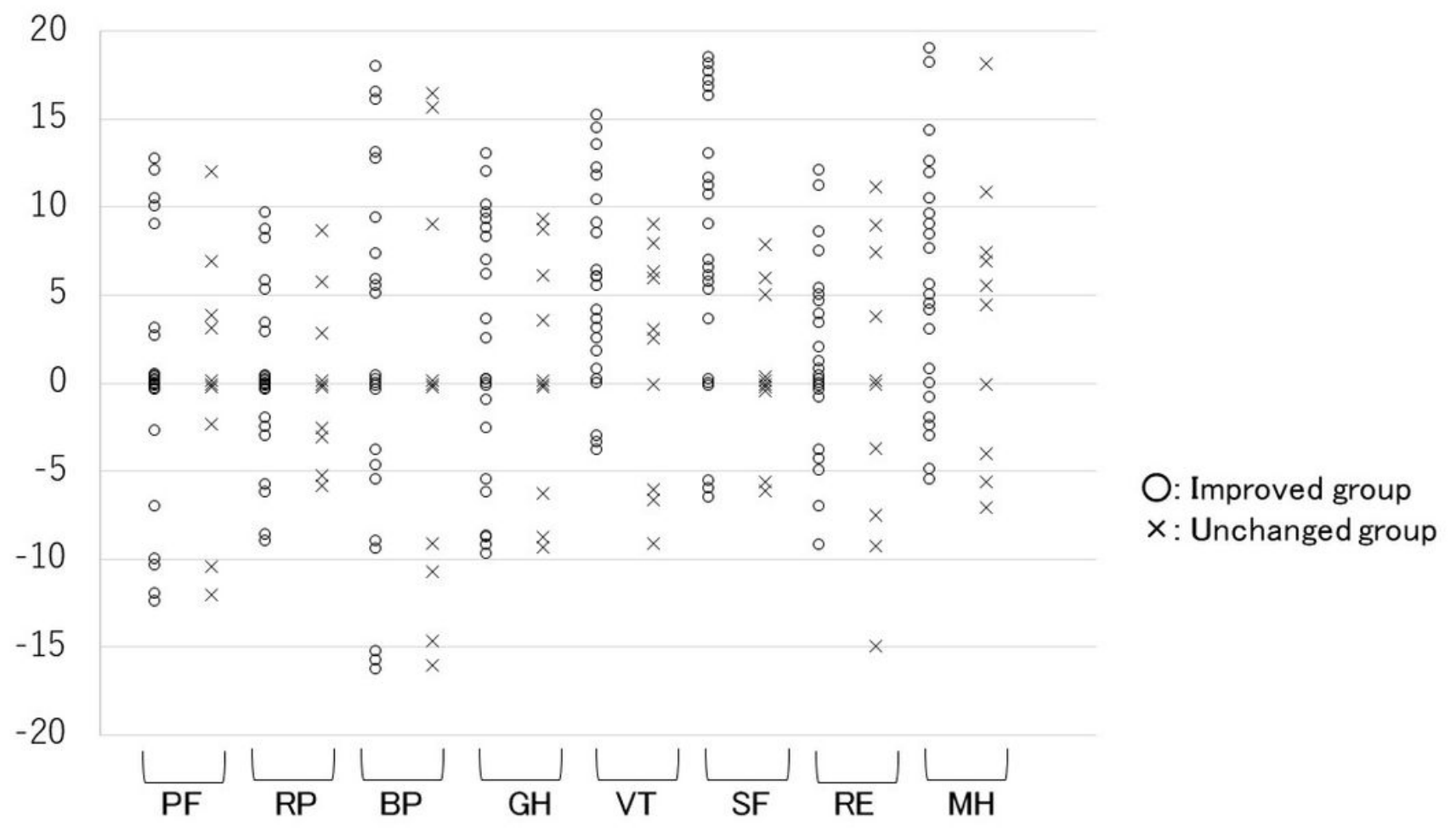

\section{Figure 3}

The distribution in the difference between the scale scores of each group before and after three months of treatment. PF, physical function; RP, role limitations because of physical health problems; BP, bodily pain; $\mathrm{GH}$, general health perception; VT, vitality; SF, social function; RE, role limitation s because of emotional problems; $\mathrm{MH}$, mental health. The VT and SF score improvement rates in the improved group were $78 \%(18 / 23)$ and $74 \%(17 / 23)$, respectively, and only 3 patients had decreased scores on both scales. 


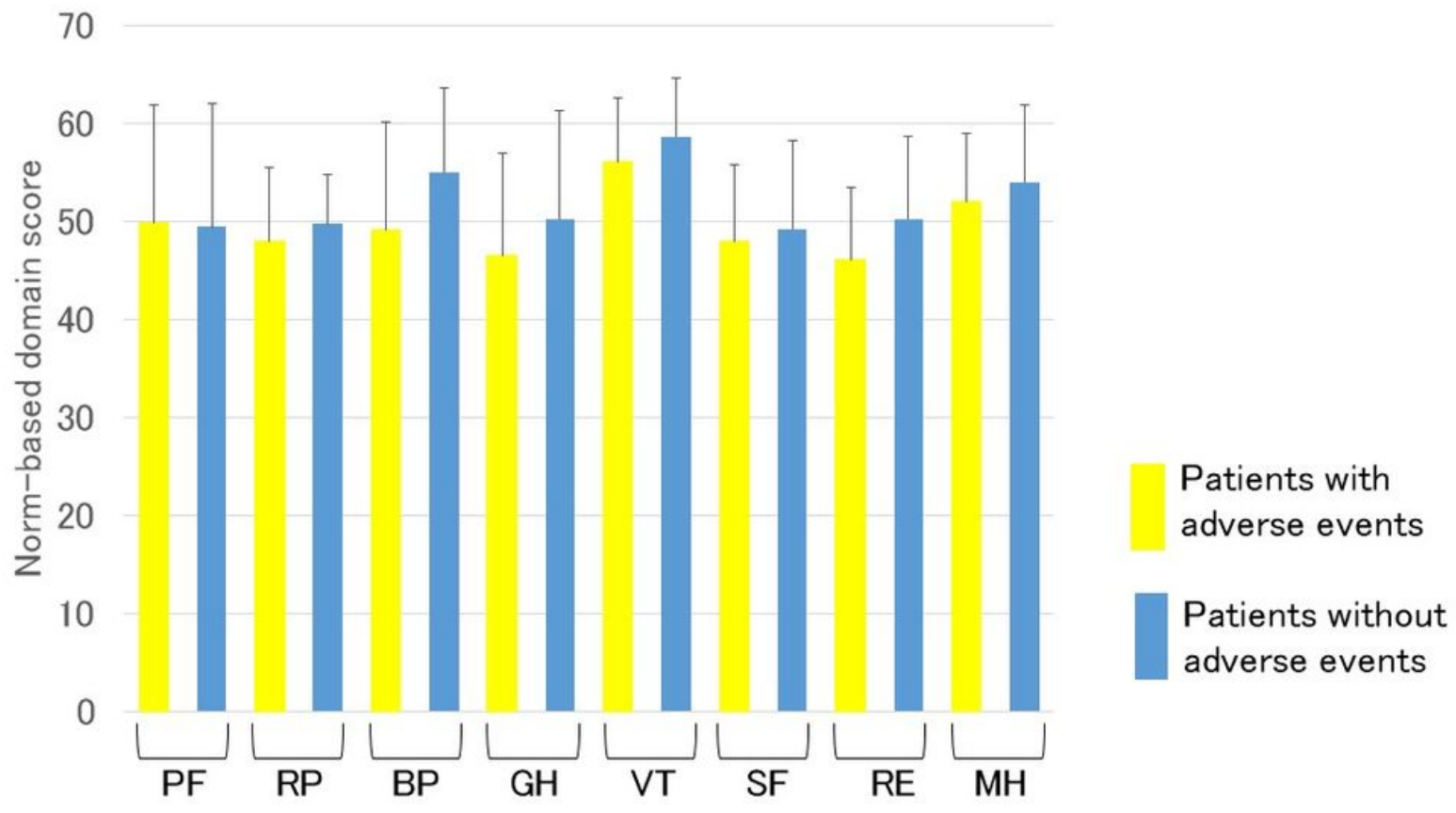

\section{Figure 4}

The comparison of the scale scores on the Medical Outcomes Study 36-Item Short Form three months after the initiation of sirolimus gel treatment for facial angiofibroma associated with tuberous sclerosis complex between patients with and without treatment-related adverse events. PF, physical function; RP, role limitations because of physical health problems; BP, bodily pain; $\mathrm{GH}$, general health perception; $\mathrm{VT}$, vitality; SF, social function; RE, role limitations because of emotional problems; $\mathrm{MH}$, mental health. There were no significant differences in any scale scores between these two groups. 